\title{
HIV-1 Infection: The Functional Importance of SDF1, CCR2 and CCR5 in Protection and Therapeutics
}

\section{Ranjeet Singh Mahla*}

Department of Biological Sciences, Indian Institute of Science Education and Research Bhopal, India

\begin{abstract}
The acute or chronic infection of HIV1 resulting to AIDS pandemics is one of the major causes of morbidity and mortality worldwide. The infection, prevalence and propagation of HIV1 depend both on adaptive mutation in virus and host genetic factors. Virus infection to human CD4+ immune cells together is assisted and restricted by various host factors. Presence of mutations in CCR2, CCR5 and CXCR4 ligands SDF1 are associated to protection against HIV1 infection and restriction to AIDS progression. Globally, individuals in various populations harbouring CCR2 (64I), CCR5 $(\triangle 32)$ and SDF1-3'A mutations are less susceptible to HIV1 infection and decipher delayed onset of AIDS. This review emphasizes the distribution and functional importance of CCR2 (64I), CCR5 $(\Delta 32)$ and SDF1 $\left(3^{\prime} A\right)$ mutations in protection against HIV1 infection. Lastly the review discuss how CCR2, CCR5 and SDF1 can be explored for development of antagonistic for protection against HIV1 infection.
\end{abstract}

Keywords: HIV1; Host genetic factors; Chemokine receptors; Therapeutics

\section{Introduction}

Infection of human immunodeficiency virus (HIV)-1, the causative agent of spectrum of disease known as acquired immuno deficiency syndrome (AIDS), is a global pandemics. The prevalence and pattern of HIV1 infection varies globally. Some countries are more affected from fatal consequences then the others and the infection prototype varies within the country itself [1]. Despite global awareness and implementation of prevention strategies, the infection of HIV1 has increased at alarming rates in different parts of the world [1]. Advancement in the field of immunology, virology and population genetics has enabled us to understand the complex biology of HIV1 infection. The prevalence of HIV1 infection and progression to AIDS depends both on virus and host genetic factors [1]. There is tropism in HIV1 infection. The M-tropic and T-tropic HIV1 mediates their infection to human CD4+ cells by viral coat proteins gp120 and gp41 respectively. For HIV1 infection the $\mathrm{G}$ protein coupled seven transmembrane $\mathrm{C}-\mathrm{C}$ chemokines receptors CCR2, CCR5, CXCR4 of host are extremely critical [2]. The R5, X4 and R5X4 strain of HIV1 exploit host CCR5, CXCR4 and both CCR5 and CXCR4 receptor respectively for their entry to host cells. The expression status of these three host factors namely CCR2, CCR5 and CXCR4 ligand SDF1 (stromal cell derived factor 1) on CD4+ immune cells decides the prevalence, propagation of HIV1 infection. Individuals among different populations harbouring CCR2 (64I), CCR5 $(\triangle 32)$ and stromal cells derived factor 1-3'A (SDF1-3'A) mutant allele are comparatively more protected against infection of $\mathrm{M}$ and $\mathrm{T}$ trophic strains of HIV1 [3]. The presence and distribution of mutant, minor and wild allele at CCR loci and their respective involvement in disease progression and resistance against infection are the key signature marks of host pathogen co-evolution. This review will discuss about distribution of CCR2 (64I), CCR5- $\triangle 32$ and SDF1-3'A, alleles in diverse populations and how much important these alleles are for their role against HIV1 infection. HIV1 infection, geographical distribution, resistance towards drugs and therapeutic has captured a massive attention of research in the field of molecular biology, epidemiology and population genetics. Lastly the review emphasizes the key question, how natural ligands (SDF1, RANTES, MIP1- $\alpha$, and MIP1- $\beta$ ) and synthetic antagonists against CCR2, CCR5 and SDF1 can prevail protection both $\mathrm{M}$ and $\mathrm{T}$ trophic HIV1.

\section{SDF1 and HIV1}

The chemokine SDF1 is natural ligand of $\mathrm{C}-\mathrm{X}-\mathrm{C}$ chemokine receptor type 4 (CXCR4) has been investigated for its inhibitory role onto entry of HIV1 into its target CD4+ cells through bindings to CXCR4 which is a co receptor for T tropic HIV1 entry. However some study shows that it can exhibit dual role as inhibitor of viral entry and stimulator for expression of proviral gene through trans activation of HIV1 long terminal repeats (LTR). There are lots of controversies over SDF1 genotypes and its association to HIV1 infection [4]. Comparative meta-analysis at 95\% confidence intervals (CI) for studies supporting and contradicting SDF1 and HIV1 association deciphered that, for healthy unexposed individuals, in comparison to WT homozygous, the pooled odds ratio (OR) for SDF1 heterozygous and SDF1 (3'A) mutant homozygous were 1.07, 1.38 and for healthy counterparts were 0.89 , 1.15 respectively. From this study it can be said that there is no statistical significance for SDF1 genotype among healthy and exposed individuals that further suggest that SDF1 genotype might have limited effect on HIV1 susceptibility [5]. A genotyping study conducted on Thais population found that presence of SDF1 (3'A) allele induces production of chemokine SDF1 that functionally competes with HIV1 virus for its binding to CXCR4 [6]. Since SDF1 is a minor allele, population with lower minor allele frequency of this allele have lower resistance against HIV1 progression after infection [6]. The SDF1 genotyping study conducted on Brazilian population covering 161 asymptomatic patients harbouring HIV1 infection (ASYMPT) versus 617 patients with AIDS status (SYMPT) shows that individuals harbouring homozygous allele for SDF1 (3'A) either does not progress to the stage of AIDS and does not die from AIDS if retroviral therapy is availed suggest that presence of

*Corresponding author: Ranjeet Singh Mahla , Department of Biological Sciences, Indian Institute of Science Education and Research (IISER), Bhopal, 462066, India, Tel: 91-9589081337; E-mail: ranjeet@iiserb.ac.in

Received October 09, 2015; Accepted December 08, 2015; Published December 14,2015

Citation: Mahla RS (2015) HIV-1 Infection: The Functional Importance of SDF1 CCR2 and CCR5 in Protection and Therapeutics. Health Care Current Reviews 3 : 150. doi: $10.4172 / 2375-4273.1000150$

Copyright: ( 2015 Mahla RS. This is an open-access article distributed under the terms of the Creative Commons Attribution License, which permits unrestricted use, distribution, and reproduction in any medium, provided the original author and source are credited. 
SDF1 3'A mutation potentially involved in protection against late stage of HIV1 stimulated disease [7]. The fact further can be supported from in vitro studies on peripheral blood mononuclear cells from ASYMPT and SYMPT patients where PBMCs from HIV1 carrier ASYMT patients express higher amount of SDF1. The MAF for indigenous Han population of china is 0.28723 for SDF1 ( 3 'A) suggests that indigenous population might have more prevalence for HIV1 infection [8]. Apart from harbouring genetic variation SDF1 is natural ligand SDF1 and being that it can competitively inhibit infection of HIV1 virus (Figure 1). Overall it can be said that population harbouring higher MAF for SDF1 (3'A) alleles produce higher SDF1 chemokines that mediates protection against HIV1 infection by competitive binding to CXCR4 receptor of host CD4+ cells.

\section{CCR2 64I and HIV1}

CCR2 located on chromosome 3 p21 is an important entry coreceptor for HIV1 virus infecting host CD4+ cells [9]. Presence of 64Valine to Isolucine mutation in the coding region of this gene has been credited for delaying disease progression that is supported by stable or decreased viral loads and showed decline in CD4+ cells populations in HIV1 infected patients. Identification and characterization of mutant 64I allele among individuals of a population has prospective for development of fusion and entry inhibiting factors [10]. Population genetics studies conducted on Kenyan population suggest that $>78 \%$ of the individual infected with HIV1 harbours V/V wild type allele where only 4 out of 118 individuals, included in study had I/I homozygous genotype, suggest that host genetic factors are very important tool in predicting the epidemiology of HIV1 prevalence and progression for healthy individuals [11]. But this is not a universal fact that CCR264I completely protects against HIV1 infection. For example the Gabon population in 1990s remained protected from HIV1 infection where neighbouring countries severely suffered from the HIV1 pandemics. Later on, genotyping of these population for CCR2 gene revealed, that there is no direct relationship between presence of CCR2 64I mutation and HIV1 infection. Rather the protection against HIV1 infection was mediated by environmental factors [12]. An independent study on indigenous Han population from China consisting of only few thousand individuals has minor allele frequency (MAF) 0.20023 where no individual are homozygous for 64I allele suggests that indigenous population might have higher prevalence of HIV1 infection [13].

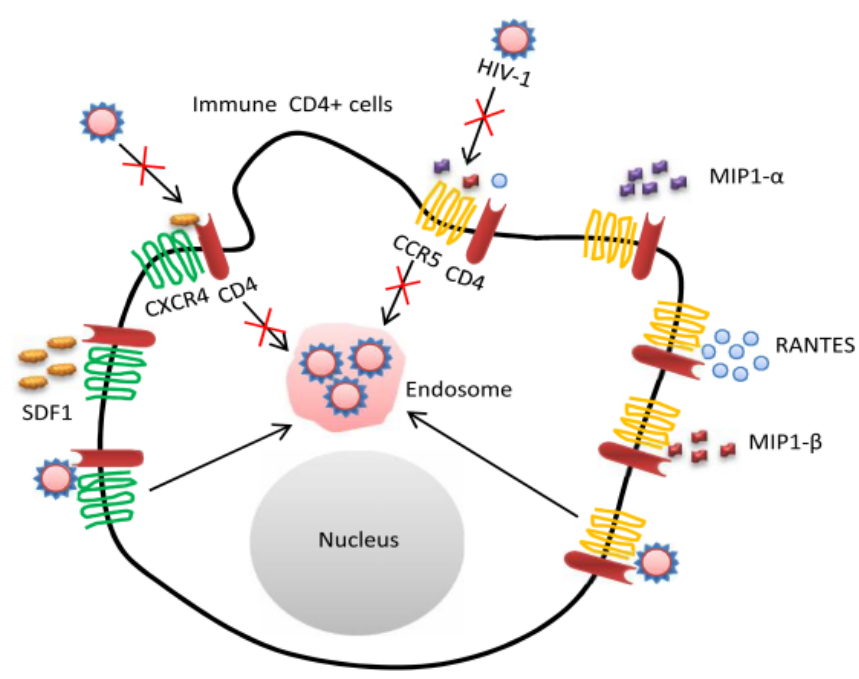

Figure 1: Natural ligand mediated inhibition of HIV1 infection.
In recent scenario analysis of global impact for $64 \mathrm{~V} / \mathrm{I}$ can be more promising then small scale studies considering that a meta-analysis of 19 of such individual genotyping studies on different populations which are descended from Africa shows that CCR2 (64I) allele among seroconverts is associated with lower risk for progression to AIDS with relative hazards $(\mathrm{RH})$ value of 0.76 and decreased risk of death with RH 0.74. In association to CCR264-I, after sero-conversion the level of HIV1 RNA (copies/ml) can be lower to $-0.14 \mathrm{log}$ but this allele had no concrete protective effect from death after development of AIDS $[10,14]$. Similar to other African populations the discordant couples of Zambian population, the HIV1 co receptor CCR2 64I haplotypes and diplotypes modulates viral load by index seropositive and HIV1 exposed seronegative [15]. In conclusion it can be said that overall distribution of CCR264I allele in a population favours protection against HIV1 infection but that is not a full proof guarantee as there are lot more environmental clues over genetic factors.

\section{CCR5 ( $\Delta 32)$ and HIV1}

Dendratic cells, Langerhans cells, macrophages and primary T cells predominantly express CCR5 [16]. This cell surface receptor located on chromosome 3p21, functions as co receptor for HIV1 entry to CD4+ cells and even since its discovery CCR5 is central to all studies on effects of host genetic factor on HIV1 infection [17]. Most of the individuals homozygous for CCR5 $(\Delta 32)$ (a deletion of 32 base pairs consisting of nucleotides 794 to 825 ) display no clinical symptoms of HIV1 infection and appear healthy. Most of the new infections of HIV1 to the host are from the R5 strains of HIV1 that exploits CCR5 for entry into macrophages and primary T cells [18]. Genetic polymorphism of CCR5 as WT and CCR5 $(\Delta 32)$ is one of the best documented studies showing how genetic polymorphism can regulate prevalence of disease in a population [19]. The homozygosity for 32 nucleotide deletion in coding region of CCR5 receptor (also known as delta32 allele of CCR5) does not produce functional protein that strongly hampers HIV1 transmission, whereas the heterozygous $\Delta 32$ allele can delay progression of HIV1 infection up to 2 years [20]. Presence of $\Delta 32$ mutation in CCR5 gene does not produce functional protein that correlates to the diminished progression of disease following a challenge with HIV1. Individuals homozygous for the allele CCR5 $(\Delta 32)$ appear to be protected from HIV infection while progression to disease is delayed in heterozygous carriers. Approximately $1 \%$ of the Caucasian population harbours the homozygous allele of CCR $5(\Delta 32)$ that provides protection against HIV1 infection In European countries the allelic frequency of CCR5 $(\Delta 32)$ is nearly $10 \%$ where $1 \%$ of the total European population is homozygous for this allele $[20,21]$. In contrast ingenious populations has lower MAF as it is observed in Chinese Han population as 0.00119 suggests that such populations might have more prevalence of HIV1 infection and lesser chance of protection from disease after infection [13]. Apart from the CCR5 $(\Delta 32)$ allele the host can regulate infection of the M tropic HIV1 through natural ligands synthesized by host immune system against CCR5 (Figure 1) that mainly includes chemokines regulated upon activation $\mathrm{T}$ cell expressed and secreted (RANTES), macrophage inhibitory protein (MIP)- $1 \alpha$ and MIP-1 $\beta$.

\section{CCR2, CCR5 and SDF1 antagonistic in HIV1 therapeutics}

Most of the antagonistic drugs are available against CCR2 and CCR5 while little information available for SDF1 targeting drugs for HIV1 infection. Drugs against CCR5 are new class of armamentarium against HIV1 infection where Maraviroc (Figure 2) is the first of the licensed drug against CCR5, modifies extracellular domains of receptor through interacting to Glu 283 by salt bridge, Thp 86 by T-shaped $\pi-\pi$ stacking and 
Citation: Mahla RS (2015) HIV-1 Infection: The Functional Importance of SDF1, CCR2 and CCR5 in Protection and Therapeutics. Health Care Current Reviews 3: 150. doi: 10.4172/2375-4273.1000150

\section{Antagonistic drugs for CCR2, CCR5 and SDF1}

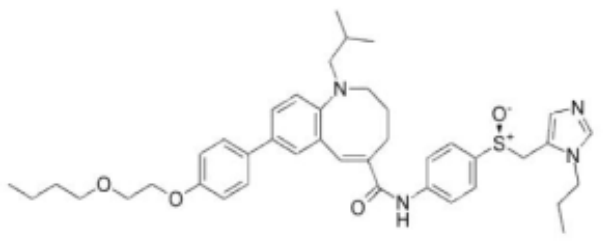<smiles>O=C1CC2(CCN(CCc3ccc(C(F)(F)F)cc3)CC2)c2ccccc2N1</smiles>

RS 102895 hydrochloride<smiles>CC(O)(O)OC(=O)OP(=O)(O)COC(CO)Cn1ccc(N)nc1=O</smiles><smiles>CCCCC(O)CCCN</smiles>

GABA<smiles>O=P(O)(O)COCCn1cnc2cncnc21</smiles><smiles>CCC(c1ccc(F)c(F)c1)n1c(C(=O)OC)c(-c2ccno2)[nH]c1=S</smiles>

JNJ 27141491<smiles>CCCCN1C(=O)C(C2CCCCC2)NC(=O)C12CCN(Cc1ccc(Oc3ccc(C(=O)O)cc3)cc1)CC2</smiles>

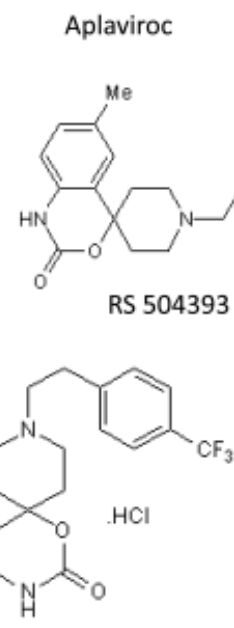

RS102895 hydrochloride

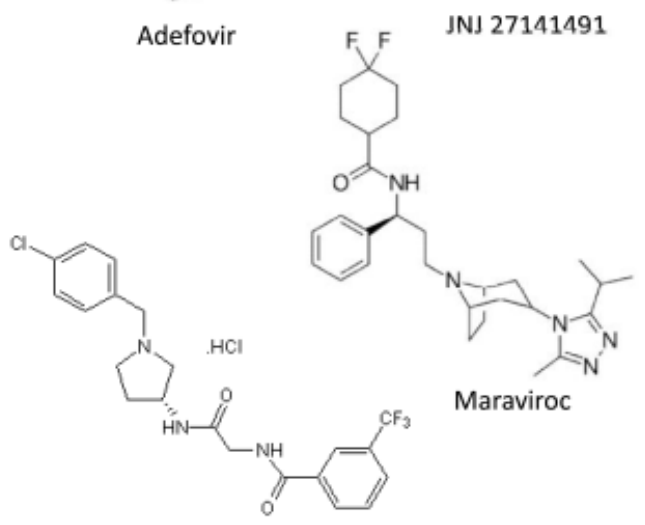

Teijin compound 1

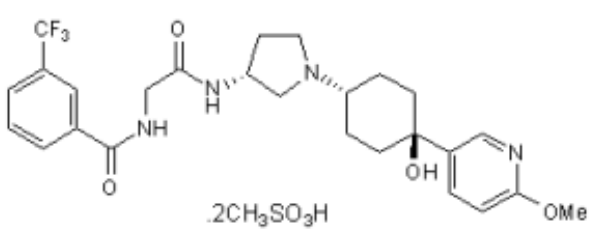

INCB 3284 demethylase

Figure 2: Antagonist drugs for CCR2, CCR5 and SDF1.

hydrophobic interaction to Phe109 [22,23]. Cenicriviroc (also known as TBR-652 is developed by Tobira Therapeutics Inc, effectively can target both CCR2 and CCR5 simultaneously through exhibition of antiretroviral and anti-inflammatory properties $[24,25]$. Acyclic nucleoside phosphonates (ANPs) is a class of antiretroviral drugs which inhibits HIV1 infection and replication through dampened the expression of CCR5 [26]. Among ANPs Cidofovir (also known as HMPC), Adefovir, Adefovir dipivoxil bis, Tenofovir and Tenofovir bis are the main compounds which affects phosphorylation status of cellular kinases leading dampened propagation of HIV1 in host cells [27]. Aplaviroc a lead compound derived from spirodiketopiperazine interacts to CCR5 at Glu 283 by salt bridge, Thr 195 by hydroxyl group and at Ile198Thr115-Phe109 hydrophobic pocket by cyclohexyl group blocking HIV 1 R5 strains infection, but unfortunately this compound showed liver toxicity in primary trials and discontinued from further modifications [23]. Although the structure of these antagonist is different from each other but still all of them share the same binding pocket onto CCR5 receptor with differential binding mode [28]. INCB 3284 dimesylate, BMS CCR2 22, JNJ 27141491, RS 504393, Teijin compound 1, RS 102895 hydrochloride, are selective CCR2 antagonist (Figure 1), where INCB 3284 dimesylate and JNJ 27141491 are orally bio available for their potency [29-33]. Gamma amino butyric acid (Table 1) is a potent inhibitor for SDF1 and it is explored well for its efficacy for inhibition of migration of CD133+ haematopoietic stem cells and progenitor cells but the same will be effective for HIV 1 treatment or not is not explored well. A new class of CCR5 antagonist INCB009471 developed by Incyte (USA) effectively reduces the infection of four established clades of HIV1 and antiretroviral resistant viruses with physiologically normal CD4+ T cells count and reduced viral copies [34]. Furthermore it does not have any observed side effects but still there is a long leap for FDA approval for HIV1 therapeutics [35]. Monoclonal antibody PRO 140 against CCR5 can exhibit dose dependent antiviral activity but RCT and quasi RT comparing the potency are not sufficient for suggesting its therapeutics application, until unless large scale placebo studies are conducted on different populations [36]. Combination of Maraviroc with FLSC IgG1 can enhance binding of this antibody to CCR5 suggest that antagonists combined with antibody can exhibit stronger antiviral activity [37]. From these facts it can be said that CCR2, CCR5 and SDF1 are emerging targets for development of new generation of drugs for protection against HIV1 infection.

\section{Summary And Future Directions}

Globally the infection of HIV1 R5 or X4 or MDR strains is a major threat to human health, needs special attention for therapeutic advancement and development of positive attitude at community level for belief of survival among AIDS patients. This review have emphasized 
Citation: Mahla RS (2015) HIV-1 Infection: The Functional Importance of SDF1, CCR2 and CCR5 in Protection and Therapeutics. Health Care Current Reviews 3: 150. doi: 10.4172/2375-4273.1000150

Page 4 of 5

\begin{tabular}{|c|c|c|c|}
\hline Drug name & Target & Mode of action/efficacy & References \\
\hline Cenicriviroc & CCR2 \& CCR5 & Receptor antagonistic \& Anti-retroviral and anti-inflammatory & $\begin{array}{l}\text { Klibanov et al } 2010 \text { \& } \\
\text { Zhang et al } 2015\end{array}$ \\
\hline Maraviroc & CCR5 & Receptor antagonistic, Modifies extracellular domain of CCR5 & Roche et al 2015 \\
\hline Aplaviroc & CCR5 & CC Receptor antagonistic & Kondru et al 2008 \\
\hline Acyclic nucleoside phosphonates & CCR5 & CC Receptor antagonistic, down regulates expression of CCR5 & Potměšil et al 2015 \\
\hline INCB009471 & CCR5 & Receptor antagonistic & Cohn et al 2007 \\
\hline RS 102895 hydrochloride & CCR2 & CC Receptor antagonistic & Mirzadegan et al 2000 \\
\hline BMS CCR2 22 & CCR2 & CC Receptor antagonistic & Cherney et al 2008 \\
\hline INCB 3284 dimesylate & CCR2 & CC Receptor antagonistic \& orally bio available & Xue et al 2011 \\
\hline JNJ 27141491 & CCR2 & CC Receptor antagonistic \& orally bioactive & Buntinx et al 2008 \\
\hline RS 504393 & CCR2 & CC Receptor antagonistic & Mirzadegan et al 2000 \\
\hline Teijin compound 1 & CCR2 & CC Receptor antagonistic & Moree et al 2004 \\
\hline GABA & SDF1 & Chemokine antagonistic & Seidel et al 2007 \\
\hline PR0-140 & CCR5 & Mab blocks CCR5 & Li et al 2010 \\
\hline FLSC IgG1 & CCR5 & Mab blocks CCR5 & Latinovic et al 2015 \\
\hline
\end{tabular}

Table 1: CCR2, CCR5 and SDF1 antagonistic drugs.

the distribution and functional importance of CCR2 $(64 \mathrm{I})$, CCR5 $(\Delta 32)$ and SDF1 (3'A) alleles and their protective role against HIV1 infection. The availability of cost effective drugs, social and religious stigma, sex education and genetic transmission of HIV1 infection are major hurdle for cure and protection against this deadliest virus. This review points outs the importance of natural ligand SDF1, RANTES, MIP1- $\alpha$, and MIP1- $\beta$ in AIDS therapeutics and how far the research exploring antagonistic against natural variants of CCR2, CCR5 and SDF1 have moved so far for protection against HIV1 tropism. The emotional care and removal of stigma of AIDS patient can't be tartan without lively participation of family and community where individuals are affected from HIV1 infection. In this direction many of the community oriented research organizations like YRG cares in India and others from different parts of the world had strong stand in educating people about HIV1 infection threats and providing motivational support to HIV1 patients for procuring treatment and considering the importance of life and survival. For eradication of global HIV1 pandemics there is an immediate need of development of new class of drugs against single or multi drug resistant strains of HIV1. Identification of new host genetic factors as markers for diagnosis and prognosis of HIV1 infection, prediction of infection prevalence and pandemics through viral and host genetic factors can be the potential tools for development of new class of drugs, where all these things can be combined for to host immunological protection profile for commencing personalized therapy of both ASYMPT and SYMPT patients (Figure 2).

Administration of human host with chemically synthesized antagonist drugs blocks functioning of chemokine receptor CCR2 and CCR5. This blocking inhibits entry of HIV1 to CD4+ immune cells (macrophages, primary $\mathrm{T}$ cells and Langerhans cells) that ultimately provides protection from HIV1 infection and protects host from infection (Figure 1).

Chemokine receptors CCR5 and CXCR4, localized on plasma membrane of CD4+ Primary T cells, macrophages and Langerhans cells mediates entry of HIV1 to these cells. Natural ligands MIP1 $\alpha$, MIP1- $\beta$ and RANTES which are natural ligands for CCR5, indirectly blocks receptor mediated entry of M- tropic HIV1 virus while SDF1 the natural ligand for CXCR4 inhibits infection of T- tropic HIV1 though unavailability of receptor for viral infection (Table1).

\section{Acknowledgement}

Mrs Sarita Kumari, Department of Yoga, Barkatullah University, Bhopal and Dr. Anuradha Reddy from Centre for Cellular and Molecular Biology, Hyderabad, India are acknowledged for their useful comments on the manuscript.

\section{Conflicts of Interest}

Author declares no conflicts of interest

\section{References}

1. Cohen MS, Hellmann N, Levy JA, DeCock K, Lange J (2008) The spread, treatment, and prevention of HIV-1: evolution of a global pandemic. J Clin Invest 118: 1244-1254.

2. Michael NL, Louie LG, Rohrbaugh AL, Schultz KA, Dayhoff DE, et al. (1997) The role of CCR5 and CCR2 polymorphisms in HIV-1 transmission and disease progression. Nat Med 3: 1160-1162.

3. de Silva E, Stumpf MP (2004) HIV and the CCR5-Delta32 resistance allele. FEMS Microbiol Lett 241: 1-12.

4. Marechal V, Arenzana-Seisdedos F, Heard JM, Schwartz O (1999) Opposite effects of SDF on human immunodeficiency virus type 1 replication. J Virol 73 3608-3615.

5. Liu S, Zhu H (2011) Association between polymorphism of SDF1 (CXCL12) gene and HIV-1 susceptibility: a meta-analysis. Curr HIV Res 9: 112-119.

6. Tiensiwakul P (2004) Stromal cell-derived factor (SDF) 1-3'A polymorphism may play a role in resistance to HIV-1 infection in seronegative high-risk Thais. Intervirology 47: 87-92.

7. Reiche EM, Watanabe MA, Bonametti AM, Morimoto HK, Morimoto AA et al. (2006) Stromal cell-derived factor 1 (SDF1) genetic polymorphism in a sample of healthy individuals, seronegative individuals exposed to human immunodeficiency virus type 1 (HIV-1) and patients infected with HIV-1 from the Brazilian population. Int J Immunogenet. 33:127-133.

8. Wang JF, Park IW, Groopman JE (2000) Stromal cell-derived factor-1alpha stimulates tyrosine phosphorylation of multiple focal adhesion proteins and induces migration of hematopoietic progenitor cells: roles of phosphoinositide-3 kinase and protein kinase C. Blood 95: 2505-2513

9. Al-Abdulhadi SA, Al-Rabia MW (2010) Linkage and haplotype analysis fo chemokine receptors clustered on chromosome 3 p21.3 and transmitted in family pedigrees with asthma and atopy. Ann Saudi Med 30: 115-22.

10. Burton CT, Gotch FM, Imami N (2005) CCR2/64I mutation detection in a HIV-1positive patient with slow CD4 T-cell decline and delay in disease progression. Int J STD AIDS 16: 392-394.

11. Wachira D, Lihana R1, Okoth V1, Maiyo A1, Khamadi SA1 (2015) Chemokine Coreceptor-2 Gene Polymorphisms among HIV-1 Infected Individuals in Kenya. Dis Markers 2015: 952067.

12. Malhotra R, Hu L, Song W, Brill I, Mulenga J, et al. (2011) Association of chemokine receptor gene (CCR2-CCR5) haplotypes with acquisition and control of HIV-1 infection in Zambians. Retrovirology 8: 22.

13. Wang F, Jin L, Lei Z, Shi H, Hong W et al. (2000) [Distribution of HIV resistance CCR5-delta 32, CCR2-64 I and SDF1-3'A alleles and their polymorphisms in the Han population in China]. Zhonghua Liu Xing Bing Xue Za Zhi 21: 256-260.

14. loannidis JP, Rosenberg PS, Goedert JJ, Ashton LJ, Benfield TL, et al. (2001) 
Citation: Mahla RS (2015) HIV-1 Infection: The Functional Importance of SDF1, CCR2 and CCR5 in Protection and Therapeutics. Health Care Current Reviews 3: 150. doi: 10.4172/2375-4273.1000150

Effects of CCR5-Delta32, CCR2-64I, and SDF-1 3'A alleles on HIV-1 disease progression: An international meta-analysis of individual-patient data. Ann Intern Med 135: 782-795.

15. Malhotra R, Hu L, Song W, Brill I, Mulenga J et al. (2011) Association of chemokine receptor gene (CCR2-CCR5) haplotypes with acquisition and control of HIV-1 infection in Zambians. Retrovirology 8: 22.

16. Lederman MM, Penn-Nicholson A, Cho M, Mosier D (2006) Biology of CCR5 and its role in HIV infection and treatment. JAMA 296: 815-826.

17. Liu R, Paxton WA, Choe S, Ceradini D, Martin SR, et al. (1996) Homozygous defect in HIV-1 coreceptor accounts for resistance of some multiply-exposed individuals to HIV-1 infection. Cell 86: 367-377.

18. Carrington M, Dean M, Martin MP, O'Brien SJ (1999) Genetics of HIV-1 infection: chemokine receptor CCR5 polymorphism and its consequences. Hum Mol Genet 8: 1939-1945.

19. Mummidi S, Ahuja SS, Gonzalez E, Anderson SA, Santiago EN et al. (1998) Genealogy of the CCR5 locus and chemokine system gene variants associated with altered rates of HIV-1 disease progression. Nat Med 4:786-793.

20. Kostrikis LG, Huang Y, Moore JP, Wolinsky SM, Zhang L et al. (1998) A chemokine receptor CCR2 allele delays HIV-1 disease progression and is associated with a CCR5 promoter mutation. Nat Med 4: 350-353.

21. Samson M, Libert F, Doranz BJ, Rucker J, Liesnard C et al. (1996) Resistance to HIV-1 infection in caucasian individuals bearing mutant alleles of the CCR-5 chemokine receptor gene. Nature 382: 722-725.

22. Roche M, Borm K, Flynn JK, Lewin SR, Churchill MJ, et al. (2015) Molecular Gymnastics: Mechanisms of HIV-1 Resistance to CCR5 Antagonists and Impact on Virus Phenotypes. Curr Top Med Chem

23. Kondru R, Zhang J, Ji C, Mirzadegan T, Rotstein D, et al. (2008) Molecular interactions of CCR5 with major classes of small-molecule anti-HIV CCR5 antagonists. Mol Pharmacol 73: 789-800.

24. Klibanov OM, Williams SH, Iler CA (2010) Cenicriviroc, an orally active CCR5 antagonist for the potential treatment of HIV infection. Curr Opin Investig Drugs 11: $940-950$

25. Visseaux B, Charpentier C, Collin G, Bertine M, Peytavin G et al. (2015) Cenicriviroc, a Novel CCR5 (R5) and CCR2 Antagonist, Shows In Vitro Activity against R5 Tropic HIV-2 Clinical Isolates. PLoS One 10:e0134904.

26. PotmÄ $>\AA_{i} i l$ P, HolÃ̄1/2 A, ZÃdek Z (2015) Influence of Acyclic Nucleoside Phosphonate Antivirals on Gene Expression of Chemokine Receptors CCR5 and CXCR4. Folia Biol (Praha) 61: 1-7.
27. De Clercq E (2003) Clinical potential of the acyclic nucleoside phosphonates cidofovir, adefovir, and tenofovir in treatment of DNA virus and retrovirus infections. Clin Microbiol Rev 16: 569-596.

28. Wang T, Duan Y (2008) Binding modes of CCR5-targetting HIV entry inhibitors: partial and full antagonists. J Mol Graph Model 26:1287-1295.

29. Buntinx M, Hermans B, Goossens J, Moechars D, Gilissen RA, Doyon J, et al. (2008) Pharmacological profile of JNJ-27141491 [(S)-3-[3,4-difluorophenyl)propyl]-5-isoxazol-5-yl-2-thioxo-2,3-dihydro-1H-imida zole-4-carboxyl acid methyl ester], as a noncompetitive and orally active antagonist of the human chemokine receptor CCR2. J Pharmacol Exp Ther 327:1-9.

30. Cherney RJ, Mo R, Meyer DT, Nelson DJ, Lo YC et al. (2008) Discovery of disubstituted cyclohexanes as a new class of CC chemokine receptor 2 antagonists. J Med Chem 51:721-724

31. Mirzadegan T, Diehl F, Ebi B, Bhakta S, Polsky I et al. (2000) Identification of the binding site for a novel class of CCR2b chemokine receptor antagonists: binding to a common chemokine receptor motif within the helical bundle. J Bio Chem 275 : 25562-255671.

32. Moree WJ, Kataoka K, Ramirez-Weinhouse MM, Shiota T, Imai M et al. (2004) Small molecule antagonists of the CCR2b receptor. Part 2: Discovery process and initial structure-activity relationships of diamine derivatives. Bioorg Med Chem Lett 14: 5413-5416.

33. Xue CB, Feng H, Cao G, Huang T, Glenn J, et al. (2011) Discovery of INCB3284, a Potent, Selective, and Orally Bioavailable hCCR2 Antagonist. ACS Med Chem Lett 2: 450-454.

34. Seidel J, Niggemann B, Punzel M, Fischer J, Zanker KS (2007) The neurotransmitter GABA is a potent inhibitor of the stromal cell-derived factor1alpha induced migration of adult CD133+ hematopoietic stem and progenitor cells. Stem Cells Dev 16:827-836

35. Cohen C, DeJesus E, Mills A, Pierone G, Kumar JP (2007) Potent Antiretrovira Activity of the Once-Daily CCR5 Antagonist INCB009471 Over 14 Days of Monotherapy. 4th IAS Conference on HIV Pathogenesis, Treatment and Prevention.

36. Li L, Lee S, Wen Y, Lin C, Wan D et al. (2010) Antiretroviral Therapy Adherence among Patients living with HIVIAIDS in Thailand. Nurs Health Sci 12: 212-220

37. Latinovic OS, Zhang J, Tagaya Y, DeVico AL, Fouts TR et al (2015) Synergistic inhibition of R5 HIV-1 by the fusion protein (FLSC) IgG1 Fc and Maraviroc in primary cells: Implications for prevention and treatment. Curr HIV Res Epub ahead of print. 